\title{
ENERGY EFFICIENCY IN FILE TRANSFER ACROSS WIRELESS COMMUNICATION
}

\author{
Snigdhamayee Biswal \\ Bharath University \\ Chennai, India
}

\author{
A.Agal Veena \\ Bharath University \\ Chennai, India
}

\author{
Shilpi Kumar \\ Bharath University \\ Chennai, India
}

\author{
G.Michael \\ Bharath University \\ Chennai, India
}

\begin{abstract}
The key idea of our Energy Efficiency management is to use the exchange between energy consumption vs the gain in responsibility, timeliness, and security to maximize the system helpful time period. we tend to formulate the exchange as Associate in Nursing optimization downside for dynamically crucial the most effective redundancy level to use to multipath routing for intrusion tolerance so the question response success likelihood is maximized whereas prolonging the helpful time period. Moreover, we think about this optimization downside for the case during which a voting-based distributed intrusion detection formula is applied to sight and evict malicious nodes during a HWSN. we over see to develop a novel likelihood model to investigate the most effective redundancy level in terms of path redundancy and supply redundancy, further because the best intrusion detection settings in terms of the amount of voters and the intrusion invocation interval below that the time period of a HWSN is maximized. we over see to then apply the analysis results obtained to the planning of a dynamic redundancy management formula to identify and apply the most effective style parameter settings at runtime in response to environmental changes, to maximize the HWSN lifetime.
\end{abstract}

Keywords: Intrusion detection system (IDS), multipath routing, fault tolerance, trust management, heterogeneous WSN (HWSN).

\section{INTRODUCTION:}

Advances in wireless communication and miniature electronics have enabled the Development of small, lowcost, low-power sensor nodes (SNs) with sensing and Communication capabilities [1],[2]. Therefore, the issues of Wireless Sensor Networks (WSNs) have become popular research subjects. WSN is infrastructure based network, and through the mass deployment of SNs, a WSN is formed. The major function of WSN is to collect and monitor the related information which about the specific environment. The SNs detect the surrounding environment or the given target and deliver the data to the sink using Wireless communication. The data is then analyzed to find out the state of the target. However, due to the design of their hardware, WSNs [3]-[7] suffer from many resources Constraints, such as low computation capability, limited memory and limited energy. Because WSNs are composed by numerous low-cost and small devices which are usually deploy to an open and unprotected area, they are vulnerable to various types of attacks. A prevention mechanism is used to counteract wellknown attacks. However, interference mechanisms cannot resist overall attacks. Therefore, the attacks area unit needed to be detected. An Intrusion Detection System (IDS) is used frequently to detect the packets in a network, and confirm whether or square measure attackers. Additionally, IDS will facilitate to develop the hindrance system through acquired natures of attack. Many wireless sensor networks (WSNs) are deployed in an unattended environment in which energy replenishment is difficult. Due to limited resources, a WSN must not only satisfy the application specific QoS requirements such as reliability, minimum delay and security, but also minimize energy consumption to prolong the system useful lifetime. Recently, prior research efforts have been made to develop network architectures and sensor hardware in order to effectively deploy WSNs for a variety of applications. However, Due to a wide diversity of WSN application requirements, a general-purpose WSN design cannot fulfill the needs of all applications. In order to attain this, it is essential to capture the impacts of network parameters on network performance with respect to application specifications. Intrusion detection (i.e., object tracking) in a WSN can be regarded as a monitoring system for detecting the intruder that is invading the network domain. Thus, it is necessary to develop the intrusion detection system (IDS) which is capable of handling more extensive malicious attacks with energy conservation mechanism to increase system lifetime. If any system in the network gets affected by malicious behaviour, the request is given to the guardian system. The patch framework is given to the affected system by the guardian system and with the help the patch framework, the malicious in the affected system is cleared[8]-[10].

\section{SYSTEM ANALYSIS}

\subsection{Existing System:}

In Existing System, effective redundancy management of a clustered HWSN to prolong its lifetime operation in the presence of unreliable and malicious nodes. We tend to address the exchange between energy consumption vs. QoS gain in responsibility, timeliness and security with the goal 
to maximize the lifetime of a clustered HWSN while satisfying application QoS requirements in the context of multipath routing. More specifically, we analyze the optimum quantity of redundancy through which data are routed to a remote sink in the presence of unreliable and malicious nodes, so that the question success probability is maximized where as maximizing the HWSN lifetime.

\subsection{Proposed System:}

In planned System, the best communications vary and communication modes were derived to maximize the HWSN period of time. In intra-cluster planning and inter-cluster multi-hop routing schemes to maximize the network period of time. They thought of a hierarchic HWSN with $\mathrm{CH}$ nodes having larger energy and process capabilities than traditional SNs. Associates is developed as an improvement drawback to balance energy consumption across all nodes with their roles. In either work cited higher than, no thought was given to the existence of malicious nodes. A two-tier HWSN with the target of maximizing network period of time whereas fulfilling power management and coverage objectives. They determined the best density magnitude relation of the 2 tier's nodes to maximize the system period of time.

\section{MODULES DESCRIPTION}

\subsection{Multipath routing:}

One path reaching the sink node or base station will increase during this module, Multipath routing is taken into account an efficient mechanism for fault and intrusion tolerance to boost knowledge delivery in WSNs. the essential plan is that the likelihood of at least as we've a lot of methods doing knowledge delivery. Whereas most previous analysis targeted on exploitation multipath routing to boost responsibility, some attention has been paid to exploitation multipath routing to tolerate business executive attacks. These studies, however, mostly unnoticed the tradeoff between QoS gain vs. energy consumption which may adversely shorten the system time period.

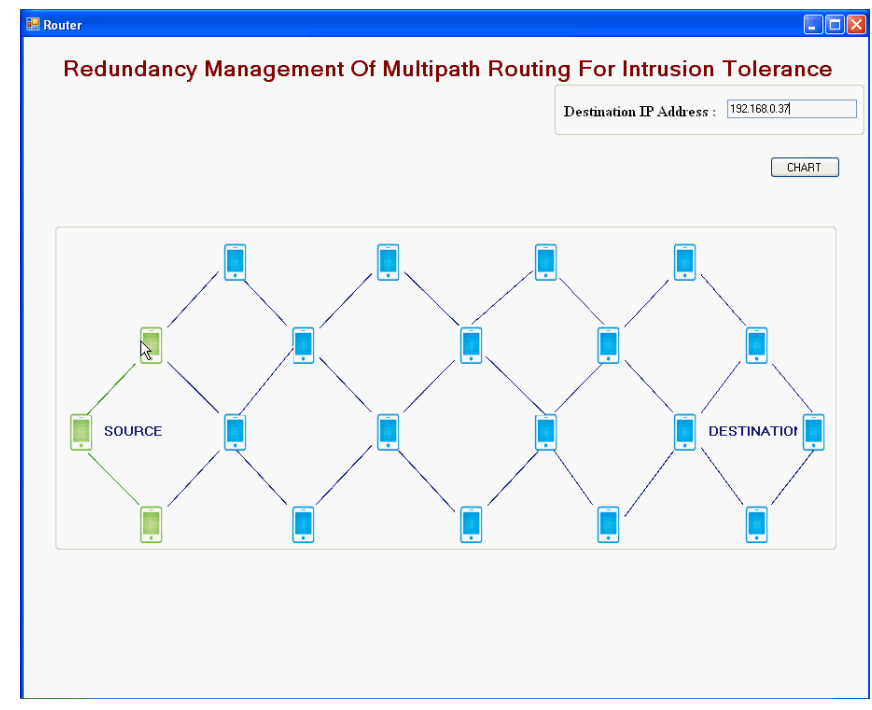

\subsection{Intrusion Tolerance:}

In these Modules, intrusion tolerance through multipath routing, there are two major issues to solve:

(1) How many paths to use and

(2) What paths to use.

Intrusion tolerance may be a fault-tolerant style approach to defensive data systems against malicious attack. Abandoning the traditional aim of preventing all intrusions, intrusion tolerance instead implies triggering mechanisms that forestall intrusions from resulting in a system security failure.

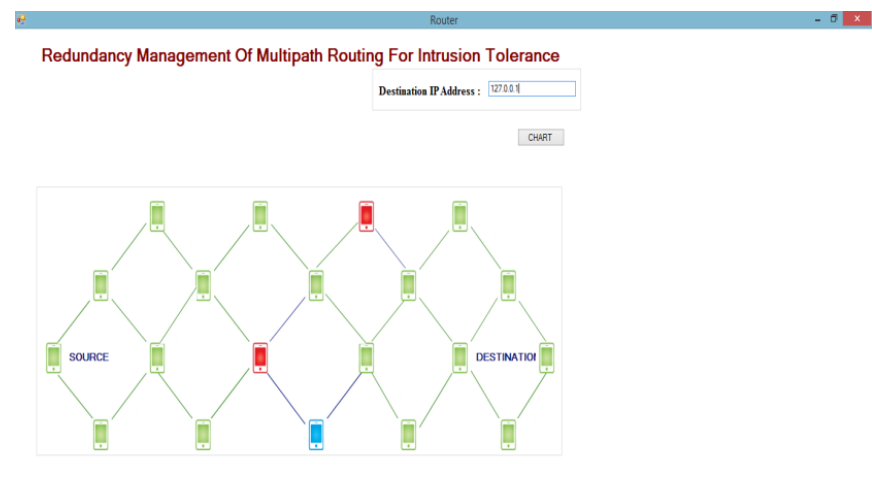

\subsection{Energy Efficient:}

In this module, there are two approaches by that energy economical IDS may be enforced in WSNs. One approach particularly applicable to flat WSNs is for AN intermediate node to feedback spite and energy standing of its neighbor nodes to the sender node (e.g., the supply or sink node) WHO will then utilize the data to route packets to avoid nodes with unacceptable spite or energy standing. Another 
approach that we tend to adopt during this paper is to use native host-based IDS for energy conservation.

\subsection{Simulation Process:}

In this module, the value of execution the dynamic redundancy management rule delineated higher than, as well as periodic bunch, periodic intrusion detection, and question process through multipath routing, in terms of energy consumption.

\section{IMPLEMENTATION IN ACTION}

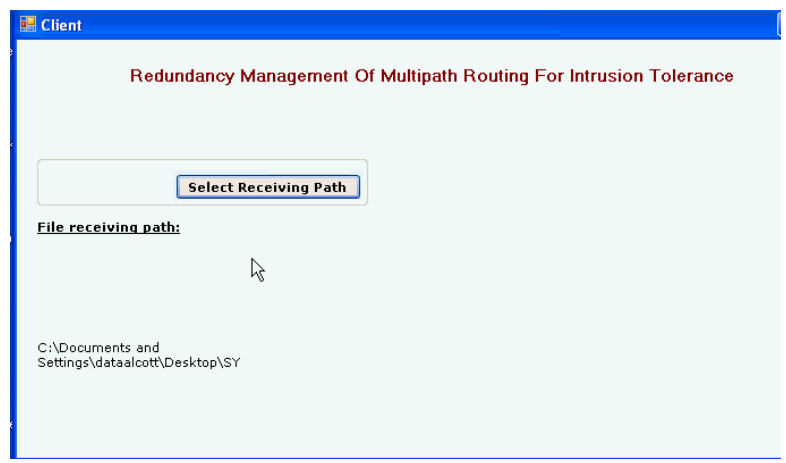

Fig. 1:Client a receive datas

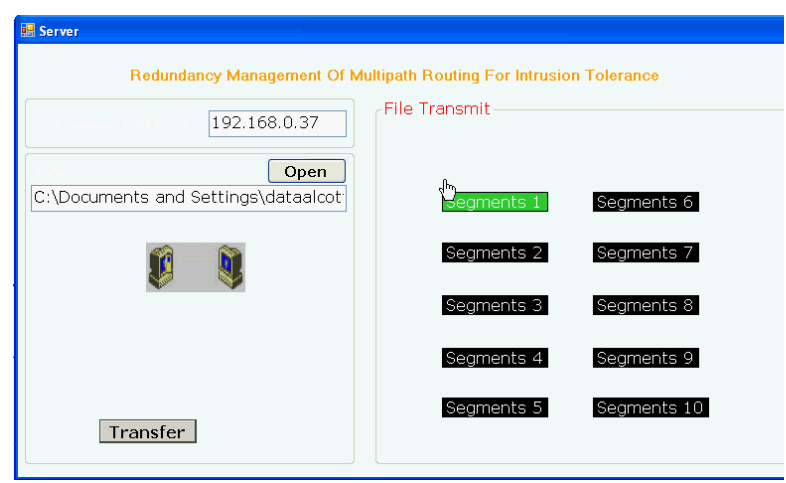

Fig. 2:Server transfers files

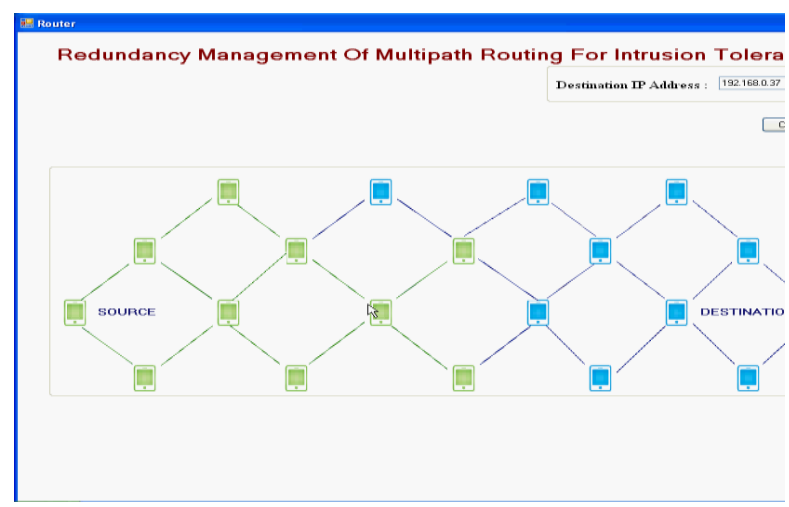

Fig. 3: Router a forward datas

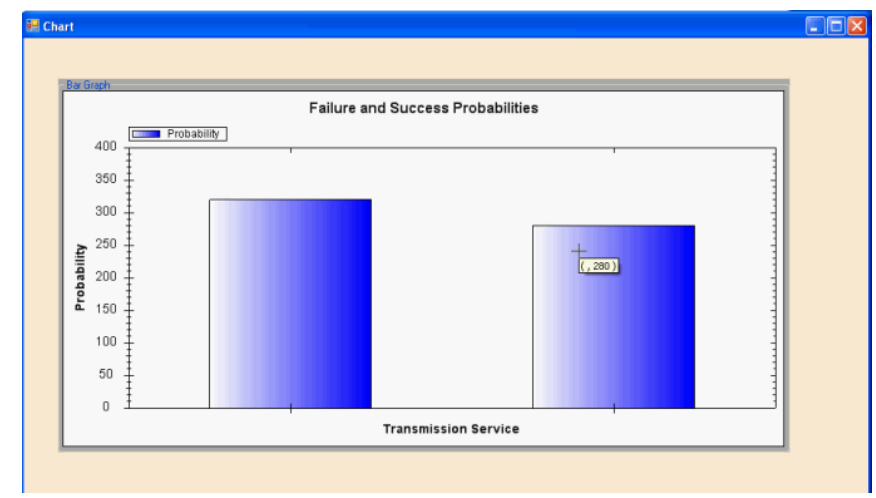

Fig.4: router transmission service

\section{CONCLUSION}

The proposed hierarchical dynamic trust management protocol for cluster-based wireless sensor networks, considering two aspects of truthfulness, namely, social truth and QoS truth. The research work will include the development of a probability model utilizing various techniques to analyze the protocol performance, and valid subjective trust against objective trust obtained based on ground truth node status. Based on the protocol the algorithm for truth-based intrusion detection will be developing using weighted choice. The algorithm will identify the best way to form trust out of social and QoS trust properties (i.e., identifying weights to assign to individual trust properties) and to assign the minimum trust threshold, in order that the performance of trust-based intrusion detection is maximized, i.e., each false positives and false negatives are minimized. Also, the research will deal with the challenging issue of providing fault tolerance in wireless device networks. Firstly a new multipath sample for heterogeneous wireless sensor networks will be define and analyzes upon various parameters. Then, propose a new fault tolerant multipath routing protocol which discovers an important number of energy node disjoint paths with the slightest overhead of one message per node. Intensive simulations will be conducted to evaluate our protocol with different scenarios, sensor nodes densities and deployment strategies.

\section{REFERENCES}

[1] O. Younis and S. Fahmy, "HEED: a hybrid, energyefficient, distributed clustering approach for ad hoc sensor networks," IEEE Trans. Mobile Comput., vol. 3, no. 4, pp. 366-379, 2004.

[2] E. Felemban, L. Chang-Gun, and E. Ekici, "MMSPEED: multipath multi-SPEED protocol for QoS guarantee of reliability and timeliness in wireless sensor networks," IEEE Trans. Mobile Comput., vol. 5, no. 6, pp. 738-754, 2006.

[3] S. Bo, L. Osborne, X. Yang, and S. Guizani, "Intrusion detection techniques in mobile ad hoc and wireless sensor networks," IEEE Wireless Commun. Mag., vol. 14, no. 5, pp. 560-563, 2007. 
International Journal of Computer Applications Technology and Research

Volume 4- Issue 4, 258 - 261, 2015, ISSN:- 2319-8656

[4] I. Krontiris, T. Dimitriou, and F. C. Freiling, "Towards intrusion detection in wireless sensor networks," in Proc. 2007 European Wireless Conf.

[5] J. H. Cho, I. R. Chen, and P. G. Feng, "Effect of intrusion detection on reliability of mission-oriented mobile group systems in mobile ad hoc networks," IEEE Trans. Reliab., vol. 59, no. 1, pp. 231-241, 2010.

[6] A. P. R. da Silva, M. H. T. Martins, B. P. S. Rocha, A. A. F. Loureiro, L. B. Ruiz, and H. C. Wong, "Decentralized intrusion detection in wireless sensor networks," in Proc. 2005 ACM Workshop Quality Service Security Wireless Mobile Netw.
[7] Y. Zhou, Y. Fang, and Y. Zhang, "Securing wireless sensor networks: a survey," IEEE Commun. Surveys \& Tutorials, vol. 10, no. 3, pp. 6-28, 2008.

[8] G. Micheal and A.R. Arunachalam "EAACK: Enhanced Adaptive Acknowledgment for MANET" Middle-East Journal of Scientific Research 19 (9), 2014.

[9] S.Parameswari, G.Michael” Intrusion Detection System in MANET : A Survey “IJETR, Vol-2, April 2014.

[10] G. Micheal "Detection of Malicious Behaviour in P2P Networks" IJETR, December 2014. 